\title{
Radiotherapy of extraosseous nasopharyngeal chordoma: A case report and literature review
}

\author{
CHI-YUAN YEH
}

\begin{abstract}
Department of Radiation Oncology, Tungs' Taichung Metroharbor Hospital, Taichung 43503, Taiwan, R.O.C.
\end{abstract}
Received September 20, 2020; Accepted February 11, 2021

DOI: $10.3892 / \mathrm{mco} .2021 .2246$

\begin{abstract}
Chordomas are slow-growing aggressive tumors that account for $1-4 \%$ of all bone tumors. The anatomical distribution of chordomas includes $50-60 \%$ in the sacrococcygeal region, $25-30 \%$ in the skull base and $15 \%$ in the mobile spine. Virchow was the first to describe and term these tumors as 'ecchordosis physaliphora' in 1857, and Muller established their notochordal origin in 1895. Extraosseous chordomas of the nasopharynx are very rare, and they exhibit similarities with other lesions of the nasopharynx, presenting as a soft tissue mass. Gross total resection combined with postoperative radiotherapy offers the best chance of long-term control. We herein present the case of a 63-year-old female patient with complaints of left temporal headaches, dizziness, left nasal obstruction, left maxillary area numbness, left ear hearing loss and swallowing difficulty. Computed tomography imaging examination revealed an $8.2 \times 3.2 \times 5.7-\mathrm{cm}$ space-occupying lesion with central necrosis in the nasopharynx and oropharynx, partially occluding the pharyngeal lumen; the mass had infiltrated the left parapharyngeal space, the left medial and lateral pterygoid muscle and the left parotid gland, with bone erosion of the left mandible. The patient was diagnosed with extraosseous chordoma of the nasopharynx, conventional type, stage IIB. The patient underwent surgery and high-dose radiotherapy and local control of the chordoma was achieved. However, the patient succumbed to a lung metastasis. The details of the case are discussed, and a review of the current medical literature is presented to provide an updated discussion on the current status of nasopharyngeal chordoma research.
\end{abstract}

\section{Introduction}

Chordomas are rare and aggressive tumors that are derived from notochordal remnants and represent $1-4 \%$ of all bone

Correspondence to: Dr Chi-Yuan Yeh, Department of Radiation Oncology, Tungs' Taichung Metroharbor Hospital, 699 Sec. 8 Taiwan Blvd., Wuqi, Taichung 43503, Taiwan, R.O.C.

E-mail: peteryeh46@gmail.com

Key words: nasopharynx, chordoma, radiotherapy, volumetric modulated arc therapy tumors. The reported incidence of chordomas ranges from 0.3-0.4 to as high as 0.84 cases per million individuals per year worldwide (1), whereas the National Cancer Database of the United States reported a total of 936 cases of cranial chordoma between 2004 and 2014 (2). The sacrococcygeal region, skull base and spine account for 50-65, 25-30 and $15-20 \%$ of chordoma localizations, respectively (3-5). The median age at diagnosis is 47-50 years and the tumor exhibits a preponderance for males $(54.8-56.2 \%)(2,6)$.

Virchow was the first to describe the presence of small nodules along the clivus in 1846, and Luschka in 1856 also described small jelly-like protrusions from the clivus (7). Virchow named these cartilaginous nodules 'ecchordosis physaliphora' in 1857, as he suggested these vacuolated mucus containing tumors were of cartilaginous origin (7). Muller established the notochordal origin of these nodules in 1895 , and Ribbert was the first to apply the term 'chordoma' in $1894(8,9)$.

Klebs reported the first case of chordoma of the clivus in 1864, in which the patient died from tumor-induced tetanic convulsions (10), and Burrow described the case of a 30-year-old male French soldier who presented with a slow growing chordoma of the skull base in 1923; the soldier experienced headaches, blindness of the left eye, left 3rd, 4 and 6th cranial nerve palsy and lower leg weakness for 3 years, and eventually died from respiratory failure. Postmortem examination revealed a $6.5 \times 5.5 \times 3.5 \mathrm{~cm}$ skull base tumor with invasion to the ethmoid and sphenoid sinus (10). The tumor was composed of alveolar cells with and abundance of cytoplasmic vacuolation due to mucin (physaliphorous cell). Seltzer (11) reported the case of a 24-year-old female patient in 1961 who experienced difficulty swallowing, neck and throat pain, and nasal speech for 6 months. Examination revealed a $4-\mathrm{cm}$ tumor in the left nasopharynx, tumor excision was performed, and pathological analysis described the tumor as containing mucus, consistent with a diagnosis of chordoma.

Chordomas usually originate from the notochordal remnants in bones, whereas extraosseous chordomas are tumors derived from soft tissue harboring notochordal remnants. Chordoma of the nasopharynx is very rare and may exhibit similarities with other lesions of the nasopharynx (12). Chordomas are amenable to complete surgical removal if there is no involvement of major neurovascular structures $(11,12)$. The silent onset of these tumors and lack of specific initial symptoms may prevent early diagnosis and, although these tumors may 
appear benign, local invasion and metastatic spread indicate that they behave more like malignant tumors (13).

Jallo et al (14) proposed a classification of chordoma based on the presence or absence of osseous origin: Type I, osseous extradural; type II, extraosseous extradural; type III, osseous intradural; and type IV, extraosseous intradural. In the present publication, the case of a type II extraosseous chordoma of the nasopharynx in an elderly female patient is presented, and a review of the current literature on chordomas is discussed.

\section{Case report}

A 63-year-old female patient was admitted to Tungs' Taichung Metroharbor Hospital on May 24, 2019. The patient experienced insidious onset of left temporal headaches and dizziness 6 months prior to admission, and subsequently complained of left nasal obstruction, left maxillary area numbness, left ear hearing loss and swallowing difficulty for 2 months prior to admission. Nasopharyngoscopy performed on 24/05/2019 revealed a left nasopharyngeal mass occupying the left Rosenmüller fossa with extension to the oropharynx. A computed tomography (CT) scan on 31/05/2019 revealed a $6-\mathrm{cm}$ left lateral nasopharyngeal heterogeneously enhanced lobulated mass in contact with the deep lobe of the left parotid gland. A biopsy and microscopic examination revealed pleomorphic adenoma, with myoepithelial cells with scant ductal epithelium in abundant chondromyxoid substance. The tumor stained positive for cytokeratin (CK)AE1/AE3 and S100, and negative for CD38, p53 and mucicarmine.

The patient developed a recurring left-sided headache, pain in the left maxillary area, left nasal obstruction and left ear deafness for 2-3 weeks; the patient was then seen on 05/09/2019 by a physician, and physical examination revealed a firm $3 \times 3-\mathrm{cm}$ indurated left aural mass and a $2 \times 2-\mathrm{cm}$ firm left submandibular mass with slight tenderness on palpation. Nasopharyngoscopy revealed a large nasopharyngeal mass completely obstructing the choanae. A subsequent CT scan on 06/09/2019 revealed an $8.2 \times 3.2 \times 5.7-\mathrm{cm}$ space-occupying lesion with central necrosis of the nasopharynx and oropharynx, partially occluding the pharyngeal lumen, and the mass infiltrated the left parapharyngeal space, the left medial and lateral pterygoid muscle and left parotid gland, along with bone erosion of the left mandible. A whole-body positron emission tomography CT scan on 14/09/2019 revealed a heterogenous ${ }^{18} \mathrm{~F}$-fluorodeoxyglucose (FDG)-avid soft tissue lesion over the left ear, nasopharynx, oropharynx, left petrous bone and left mandibular bone. The oropharyngeal tumor exhibited a maximal FDG initial and delayed standardized uptake value of 6.4 and 6.3, respectively. There was no evidence of bone, lung or liver metastases.

A transoral robotic intralesional surgery was performed on 24/09/2019 for tumor resection. The resected chordoma tissues were fixed in $10 \%$ buffered formalin for $1 \mathrm{~h}$ at $35^{\circ} \mathrm{C}$, processed and embedded in paraffin with Surgipath ${ }^{\circledR}$ Paraplast Regular (Leica Microsystems GmbH) through a Sakura Tissue-Tek VIP automated tissue processor system (Sakura Finetek USA, Inc.). All tissue blocks were then cut into 3- $\mu \mathrm{m}$ sections using a semiautomatic rotary microtome (Leica RM2245; Leica Microsystems GmbH). The histopathological sections were stained with hematoxylin solution Gill 2 (Leica Microsystems $\mathrm{GmbH}$ ) and eosin Y solution (alcoholic) (EYA999; ScyTek laboratories, Inc.) using a Sakura Tissue-Tek DRS 2000 automated stainer (Sakura Finetek USA, Inc.). Immunohistochemical staining was performed using Novocastra antibodies against CK, epithelial membrane antigen (EMA), p63, S100, Ki67 and smooth muscle actin (SMA) (product codes PA0094, PA0035, PA0103, PA0031, PA0118 and PA0943, respectively; catalog 190642 Rev B, Leica Microsystems $\mathrm{GmbH}$ ) was performed with a Leica Bond III automated stainer (Leica Microsystems GmbH).

Hematoxylin and eosin (H\&E) staining of the histopathological specimen revealed a lobular architecture with fibrous bands separating lobules with a discernible infiltrative border; the cytoarchitecture within the lobules consisted of cells forming short chords and dense epithelioid sheets of single cells within the myxoid matrix. The cells exhibited an abundance of clear to eosinophilic cytoplasm, with a bubbly, vacuolated appearance (physaliphorous cells), and occasional mitotic figures were observed (Fig. 1). On immunohistochemical staining (Fig. 2), the tumor tissues were positive for CK, EMA, p63, S100 and Ki67 (30\%), whereas SMA staining was negative. Brachyury stain was not available in our hospital. Additionally, bone, fat, muscle and vascular invasion in the tissue cross sections were also observed. The histopathological findings were consistent with a diagnosis of chordoma, conventional type (9).

Magnetic resonance imaging (MRI) on 22/10/2019 identified a recurrent huge left parapharyngeal mass lesion $(\sim 8.6 \times 4.0 \times 6.1 \mathrm{~cm})$ extending from the nasopharynx to the oropharynx, with intracranial extension to the left middle cranial fossa, left foramen ovale, left jugular foramen, left hypoglossal canal, left parotid gland and encasement of the left internal carotid artery and jugular vein (Fig. 3).

Under the clinical suspicion of nasopharyngeal chordoma pG2T2N0M0 stage IIB (15), the patient was treated with definitive radiotherapy with a cumulative dose of 70 Gy in 35 fractions between 04/10/2019 and 22/11/2019. The radiation dose to the brainstem, parotid glands, submandibular glands, optic nerves, optic chiasm, spinal cord, lens, cochlea and auditory nerves were within normal tissue tolerance doses (16). The patient was treated using the image-Guided Radiotherapy (RapidArc) technique (Varian Medical Systems, Inc.); this is a type of volumetric modulated arc therapy (VMAT) in which a triple 6-MV photon beam partial arc was delivered through a continuously arced motion of the Varian TrueBeam linear accelerator (Fig. 4). The treated tumor volume was estimated to be $213.7 \mathrm{~cm}^{3}$ using the tumor contouring function of the Varian Eclipse radiotherapy treatment planning system, version 13.0 (Varian Medical Systems, Inc.). The patient's headache and nasal obstruction were partially relieved, but the treatment course was complicated by a left otitis media with purulent discharge; this condition was managed with antibiotics. An MRI scan on 06/01/2020 revealed large areas of necrosis of the nasopharyngeal mass and slight tumor shrinkage. Further radiotherapy with 40 Gy using the VMAT technique was used to treat the residual tumor $\left(84 \mathrm{~cm}^{3}\right)$ between $11 / 02 / 2020$ and $22 / 02 / 2020$. The patient was regularly followed up in the outpatient setting up to $16 / 04 / 2020$, when the patient developed a non-productive cough. A chest X-ray revealed multiple left lower and right whole-lung metastases. As the patient developed progressive shortness of breath and moderate dry cough, 

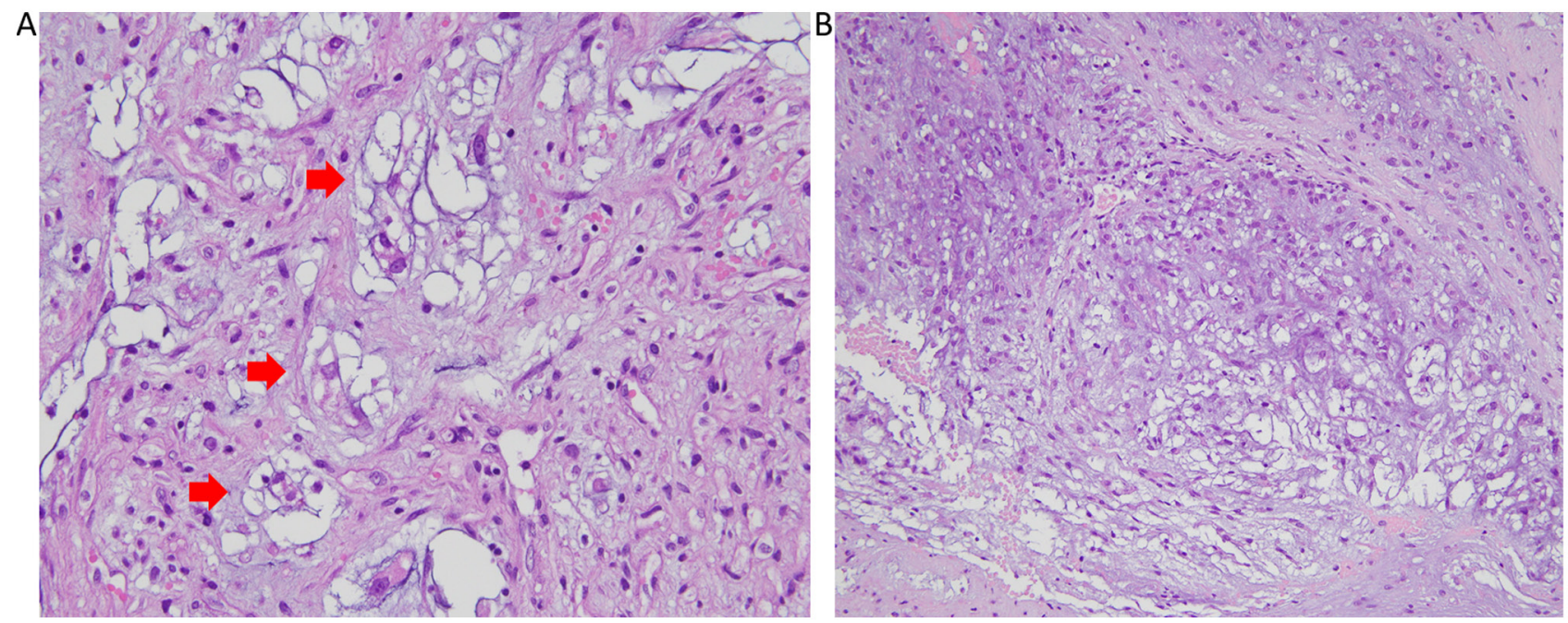

Figure 1. Histological images of the patient's tumor. (A) Tumor exhibited a bubbly vacuolated appearance of physaliphorous cells (arrows) (magnification, x400). (B) Hematoxylin and eosin staining of a cross section of the tumor, showing lobulation with extracellular myxoid matrix (magnification, x100).
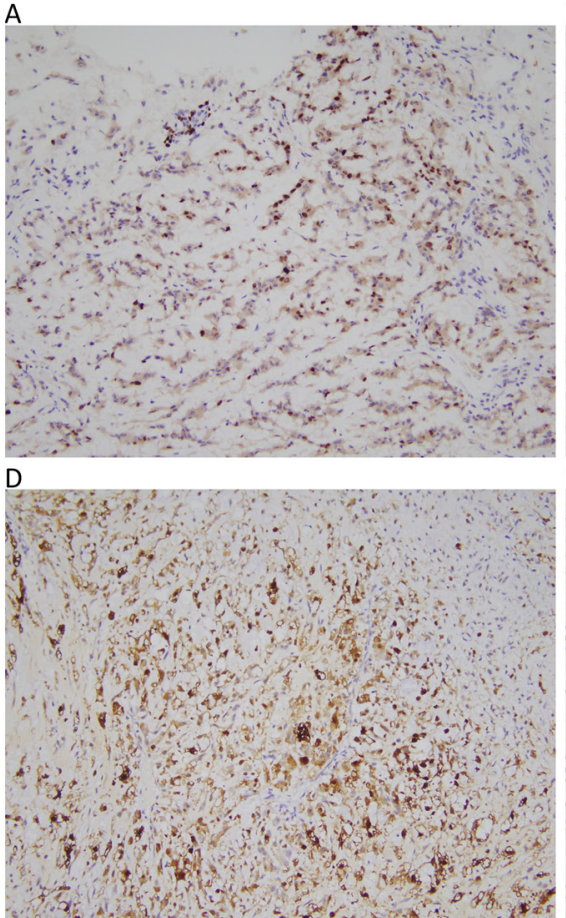
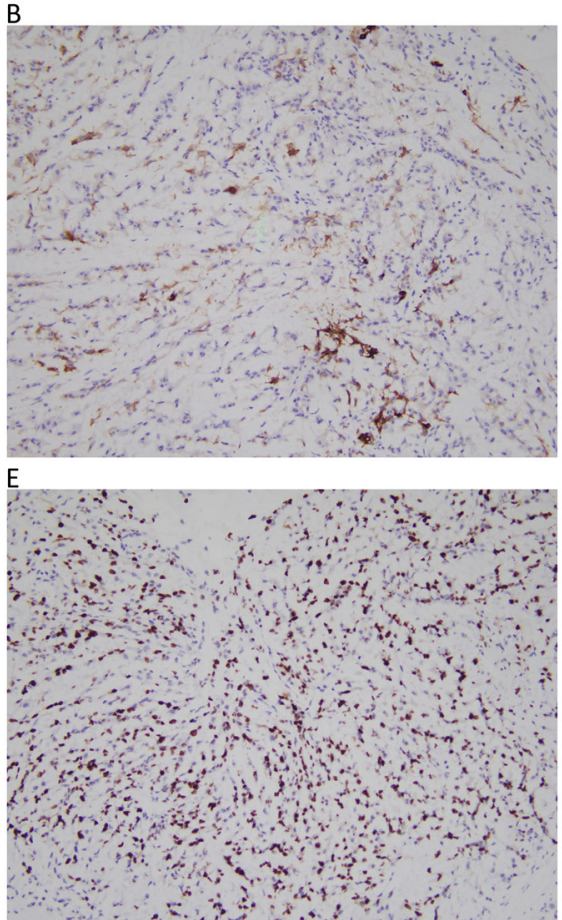
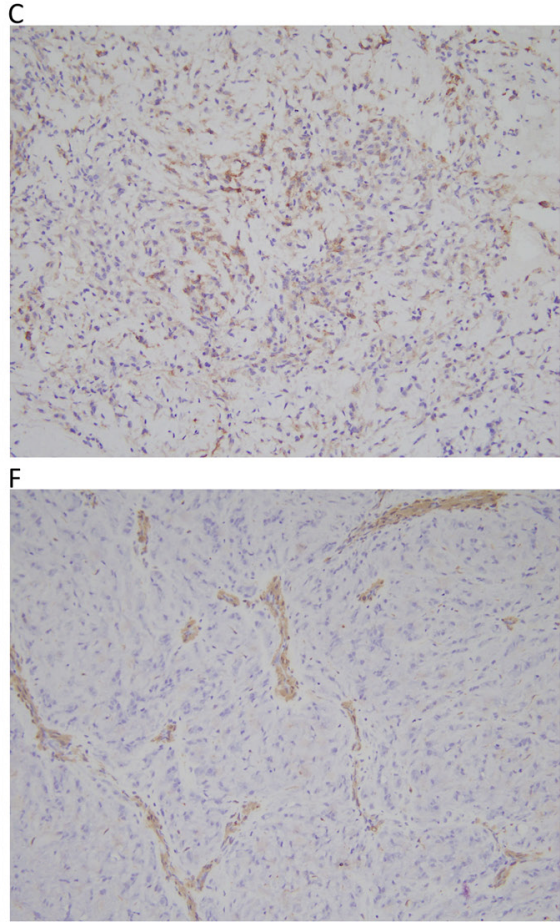

Figure 2. Immunohistochemical staining of the chordoma (magnification, x100). The tumor stained positive for (A) p63, (B) cytokeratin, (C) epithelial membrane antigen, (D) S100 and (E) Ki67, whereas it was negative for (F) smooth muscle actin.

a chest X-ray was performed on $02 / 05 / 2020$ and revealed multiple bilateral lung metastases, right-sided pneumonia and mild right pleural effusion. The patient suffered a sudden cardiac arrest and was subjected to endotracheal intubation and cardiopulmonary resuscitation; however, she succumbed to the disease on $03 / 5 / 2020$.

\section{Discussion}

Chordomas of the nasopharyngeal area represent a major challenge in terms of effective treatment due to the relative inaccessibility of the region, tumor involvement of critical neurovascular structures and invasive growth to the skull base, predisposing the tumor to local recurrence and early metastasis (12).

Chordomas are classified into three different subtypes: Conventional or classical type (accounting for the majority of the cases); chondroid type; and dedifferentiated type $(9,17)$. The different types of chordoma and their characteristics are summarized in Table I. The patient in the present case report had a conventional-type chordoma; the tumor was composed of lobules of cells arranged in cords and nests, and it is common for one tumor cells to wrap around its neighbor as if one cell is 'hugging' the other (9). The histological hallmarks of 


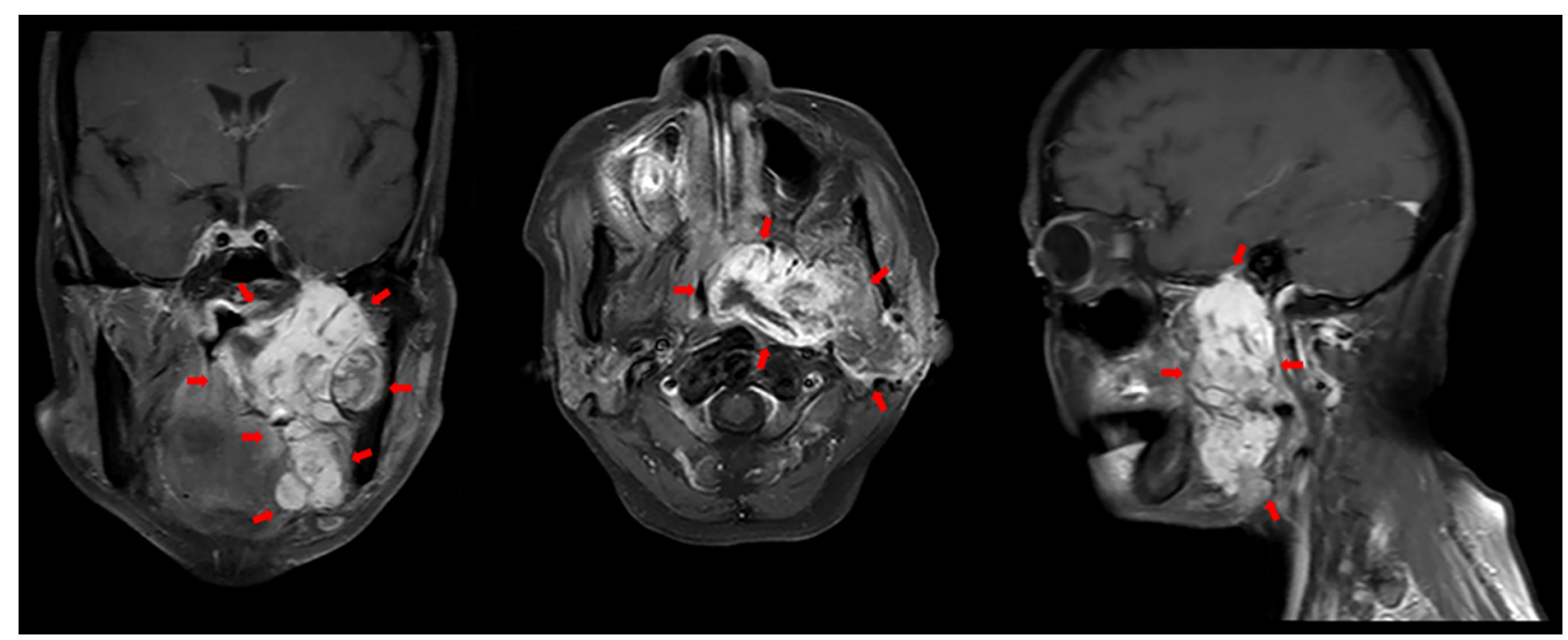

Figure 3. Gadoterate meglumine-enhanced T1-weighted magnetic resonance imaging showing a large left parapharyngeal mass extending from the nasopharynx to the oropharynx, with involvement of the skull base and left parotid gland. Red arrows are used to indicate the extent of the extraosseous nasopharyngeal chordoma.

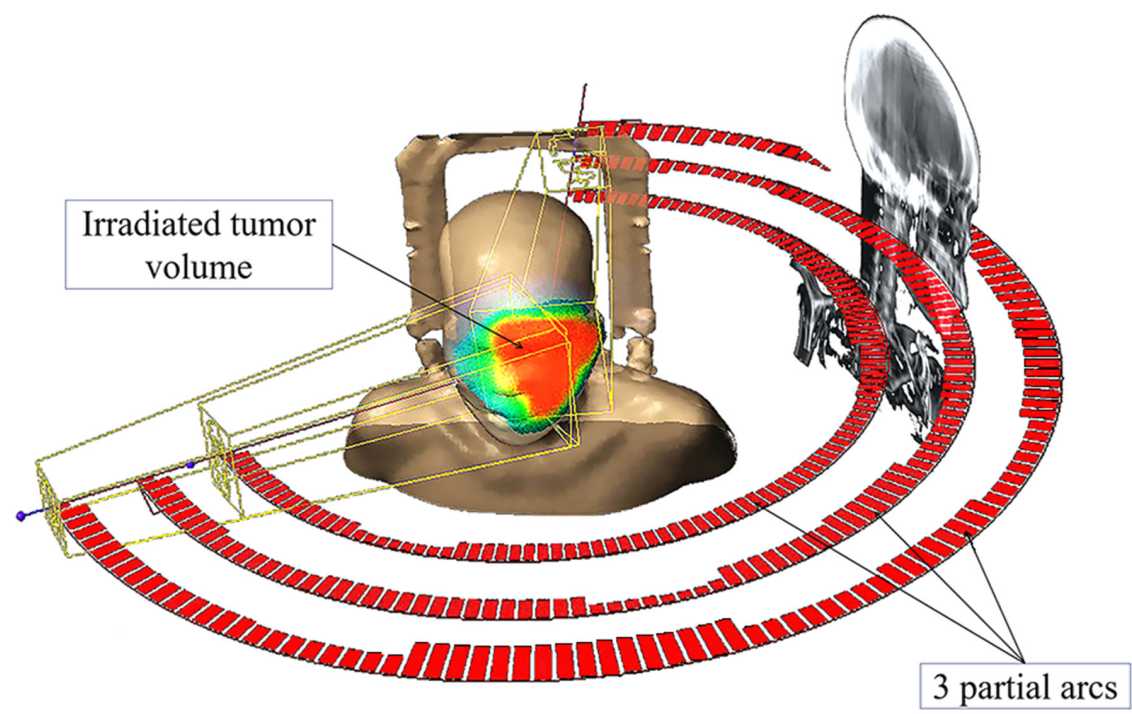

Figure 4. RapidArc volumetric modulated arc therapy using 3 partial arc linear accelerator gantry rotation for irradiation of the tumor volume.

chordoma are the large physaliphorous cells (Greek: Physalis: Bubbles, phoros: Bearing) observed on H\&E staining; these cells are characterized by the large, intracytoplasmic vacuoles embedded in a homogeneous, intercellular substance, and are arranged like beads on a string, unlike chondrosarcomas, in which the individual cells are embedded in cartilage (9). A distinguishing feature of the tumor is that it stains strongly positive for brachyury, CK, S100 and EMA, as was observed in the present case $(17,18)$. Studies have suggested that brachyury, a transcription factor encoded by the $\mathrm{T}$ (brachyury) gene for notochord development, is implicated in the initiation and progression of chordoma $(9,19)$.

Extraosseous chordoma of the nasopharynx is a rare malignancy that originates from the remnants of the notochord, a primitive tissue of embryonic origin around which the skull base and axial skeleton develop (12,20-22). Extraosseous chordomas commonly affect individuals aged
$50-60$ years $(23,24)$. The nasopharynx is, in itself, an unusual site for chordoma, accounting for only $0.2 \%$ of all nasopharyngeal neoplasms (25), and nasopharyngeal chordoma has been hypothesized to originate from the medial basal canal of the clivus, which is the notochord's cephalad exit tract from its ventral clivus location in the nasopharyngeal soft tissues $(12,21)$. To the best of our knowledge, there have only been 20 reported cases of this type of tumor in the medical literature (26). Late diagnosis is due to the non-specific and insidious onset of signs and symptoms and the slow growth rate of the tumor $(13,25)$, which is typically only detected when it has grown to a size large enough to cause a notable effect to the surrounding tissues. In the present case report, the patient presented with cranial neuropathies, pain and difficulty swallowing. Local recurrence with an incidence of $50-68 \%$ is common due to the aggressive nature of this tumor $(12,20,22,27)$, and systemic metastasis to the lungs, 
Table I. Types of chordomas.

\begin{tabular}{|c|c|c|c|}
\hline Type & Histological appearance & Gross appearance & Site of involvement \\
\hline Conventional or classical & $\begin{array}{l}\text { Lobules of cells arranged in } \\
\text { cords and nest }\end{array}$ & Soft, mucoid & Sacrum, skull base \\
\hline Chondroid & $\begin{array}{l}\text { Lacunar spaces filled with } \\
\text { neoplastic cells surrounded by } \\
\text { a solid, hyalinized matrix }\end{array}$ & $\begin{array}{l}\text { Chondroid regions merged } \\
\text { with classical component }\end{array}$ & Skull base, mobile spine \\
\hline Dedifferentiated & $\begin{array}{l}\text { Tightly packed, small, atypical } \\
\text { epithelioid cells arranged } \\
\text { in nests and sheets in a fibrous } \\
\text { background and lack of } \\
\text { physaliphorous cells }\end{array}$ & $\begin{array}{l}\text { Dedifferentiated } \\
\text { components } \\
\text { are usually distinct from } \\
\text { the areas of conventional } \\
\text { chordoma }\end{array}$ & $\begin{array}{l}\text { Sacrococcygeal area; } \\
\text { aggressive tumor with } \\
\text { systemic spread in } \\
90 \% \text { of cases }\end{array}$ \\
\hline
\end{tabular}

bones, liver and lymph nodes are found in $17.8-43 \%$ of patients with chordoma $(4,27)$.

In a retrospective study of 219 patients with chordoma, Young et al (27) found that the lung accounts for $53.8 \%$ of all metastatic sites aside from the bone, soft tissue, sternum and liver. Their study reported that cervical spine chordoma had the shortest time interval from onset to metastasis (16 months) compared with the lumbar spine (49.2 months) and sacrum (58.3 months). Young female patients were more likely to present with an aggressive chordoma of the cervical spine in the study by Young et al (27). The overall median survival was 130.4 and 159.3 months for the metastatic and non-metastatic groups, respectively. In the present case report, the patient's tumor also exhibited aggressive clinical behavior, with early lung metastasis (after 8 months) and a short survival period (9 months) after diagnosis. A study of 28 patients with skull base chordoma reported a median survival and 5-year survival rate of 4 months and $48.9 \%$, respectively, with radiotherapy alone (2).

Adverse prognostic factors include subtotal resection, dedifferentiated histological subtype, age $>40$ years, a history of prior treatment, tumor size $\geq 4 \mathrm{~cm}$, gross tumor volume $>25 \mathrm{~cm}^{3}$, pharyngeal involvement, optic nerve and brainstem compression, none or low-dose radiation therapy and female sex (1). In the present case report, the patient's gross tumor volume was $213.7 \mathrm{~cm}^{3}$, she was female and aged 63 years, and the tumor extended to the oropharynx, which collectively predicted a poorer prognosis.

Adequate wide surgery to obtain a safe margin without penetration of the capsule is the cornerstone of successful chordoma treatment; however, this is almost impossible due to the anatomical origin and involvement of nasopharyngeal and skull base neurovascular structures. Additionally, these tumors contain gelatinous material that can spill into the resection cavity, markedly increasing the risk of recurrence. The complete resection rate is only $29-50 \%$ in some studies, and subtotal resection with residual tumor is used in order to minimize surgery-related neurological deficits $(19,24)$.

A standalone endonasal approach for chordomas of the skull base is considered as a viable alternative to open resection owning to its advantages of direct tumor exposure, minimal invasiveness and favorable cosmetic appearance. Zhang et al (28) treated 32 patients with chordomas of the skull base between July 2006 and June 2015. The surgical approach was an extended endoscopic endonasal surgery. Gross total resection, subtotal resection $(>10 \%$ and $<90 \%$ tumor mass reduction) and partial resection $(>90 \%$ tumor mass reduction) was achieved in 9 (28.1\%), $16(50.0 \%)$ and 12 (21.9\%) patients, respectively. The postoperative radiotherapy dose was 54-70 Gy (mean, 62.7 Gy). Their results revealed a 3- and 5-year progression-free survival (PFS) of 44.0 and $16.5 \%$, respectively. The 3- and 5-year overall survival (OS) rate was 79.4 and $69.5 \%$, respectively. The PFS between the surgery and radiation group and the surgery alone group exhibited no significant differences; however, there was a significant increase in OS for the surgery plus radiation group compared with the surgery alone group. It was concluded that gross total resection is associated with improved PFS and OS, and postoperative adjuvant radiation therapy is recommended to improve OS for chordomas of the skull base.

Postoperative radiotherapy with photon or proton beam currently serves an important role in chordoma management and has been reported to show a positive outcome $(2,4,13,19)$. Radiotherapy for head and neck cancer, such as in the present case report, is a challenging task due to the complex anatomy of the head and neck region, with these tumors being located in close proximity to radiation dose-limiting critical structures. These tumors behave aggressively and grow rapidly due to the rich lymphatic supply in the head and neck region, and almost always manifest at a locally advanced stage (29).

Foweraker et al (6) treated 9 adult cases of chordoma and 3 cases of chondrosarcoma of the skull base with preoperative radiotherapy of 50-60 Gy, and a postoperative boost radiotherapy of $5 \mathrm{~Gy}$, with a cumulative dose of 65 Gy delivered in 39 fractions. Local control was achieved in 11 of the 12 cases, and the 3-and 5-year cause-specific survival for the 12 patients was 88 and $75 \%$, respectively; 1 patient developed local recurrence 23 months after radiotherapy, while another patient developed lymph node metastasis 7 months after radiotherapy and succumbed to distant metastasis 20 months later. In the present case report, the patient also exhibited a similar clinical course and succumbed to the disease 7 months after radiotherapy. Thus, high-dose radiotherapy may be a viable palliative treatment option for this disease.

Image-guided intensity-modulated radiotherapy (IG-IMRT) was used to treat 24 chordomas and 18 chondro- 
sarcomas of the skull base postoperatively, as reported by Sahgal et al (30), with a median dose of 76 and 70 Gy for chordoma and chondrosarcoma, respectively. The 5-year OS and local control rate for skull base chordoma and chondrosarcoma were $85.6,65.3,87.8$ and $88.1 \%$, respectively. A total of 6 patients (14.3\%) developed radiation-induced late toxicity after a mean follow-up period of 3.2 years, including hearing loss, hypothyroidism, hypopituitarism, vestibular nerve injury and cranial nerve IV injury; these patients were treated for skull base chordoma with 78 Gy in 39 fractions. Thus, careful treatment planning can achieve acceptable local control with tolerable radiation-induced toxicity.

Gatfield et al (31) treated 28 patients with chordoma of the skull base and spine with postoperative radiotherapy; these patients were treated with IG-IMRT with a median dose of $65 \mathrm{~Gy}$ (range, 65-70 Gy) and were able to achieve a 5-year local control rate and cause-specific survival rate of 74 and $85 \%$, respectively. It was concluded that postoperative high-dose radiotherapy may result in long-term control of the disease.

Salazar Guilarte et al (22) reported the case of a 7-year-old girl with a $4 \times 3 \times 5-\mathrm{cm}$ nasopharyngeal chordoma, who first underwent transoral endoscopic resection. Subsequently, stereotactic ablative radiotherapy was used to treat the $2-\mathrm{cm}$ residual tumor with good results; dynamic IMRT was used to deliver $42 \mathrm{~Gy}$ in 4 fractions. The patient has remained disease-free 20 months after radiotherapy.

High-dose radiotherapy using modern techniques, such as the RapidArc VMAT for nasopharyngeal chordoma deliver promising results. This advanced technique has been used extensively to treat nasopharyngeal carcinoma, achieving a 3-year locoregional control rate and 3-year OS rate of 92.2 and $97.0 \%$, respectively (31). RapidArc is an advanced technique in which radiotherapy treatment is delivered using a continuous arc motion of the linear accelerator gantry, accompanied with simultaneous variation of the multileaf collimator position, gantry rotation speed and dose rate output; this technique delivers a highly conformal radiation dose to the tumor, whilst limiting the dose to the organs at risk surrounding the tumor (29).

Prognostic factors for local control in primary chordomas include tumor size, extent of resection, quality of surgery, patient age, technique and quality of radiotherapy (32).

A dose of at least 74 Gy is recommended using conventional fractionation for photon and proton therapy (PT). Target volumes should be delineated considering the primary tumor location and its recurrence sites. The high-dose volume should include any macroscopic disease as well as surgical margins, whereas the low-dose volume should encompass areas at risk of microscopic spread, skip metastases, or seeding due to previous surgical procedures (32).

Particle beam with PT or carbon ion therapy (CIT) have also been reported to obtain excellent results in terms of local control and survival; the reduced lateral scatter due to the Bragg peak offers a more conformal dose distribution when compared with photon beam radiotherapy; this will enable a higher tumoricidal radiation dose to chordomas of the skull base, without incurring any severe late neurovascular toxicities (33). Takagi et al (33) treated 24 patients with skull base chordoma with PT and CIT between April 2003 and May 2014. The radiation dose constraints of the optic nerve, chiasma, cochlea, spinal cord and brainstem were limited to $\leq 47 \mathrm{~Gy}$.
The study reported a 5-year local control, PFS and OS rates of 85,81 and $86 \%$, respectively, during a median follow-up of 71.5 months (range, 14-175 months) for all patients. Patients who received postoperative particle therapy achieved a statistically significant improvement in local control, PFS and OS. Of note, 2 patients developed brain necrosis associated with moderate cognitive and memory dysfunction, and 1 patient developed bleeding from the nasopharynx that was treated with coil embolization (33). Thus, particle beam therapy was deemed to be an effective and safe treatment for chordomas of the skull base in the postoperative setting to improve local control.

Systemic therapy is an option for unresectable, locally recurrent or metastatic chordomas. Meng et al (34) reviewed a variety of molecular target inhibitors (MTIs), such as imantinib, erlotinib, cetuximab, sorafenib, pazopanib, sunitinib, sirolimus, thalidomide, bevacizumab, gefitinib, linsitinib and everolimus, which were included in 32 clinical trials.

Imatinib is a tyrosine kinase inhibitor targeting platelet-derived growth factor $b$, and it is the most extensively studied MTI for the treatment of chordomas. Overexpression and activation of platelet-derived growth factor receptor $b$ is hypothesized to play a role in the growth of chordomas. Across four studies, with a total of 181 chordoma patients treated with imatinib, partial response was achieved in 4 patients $(2.2 \%)$, whereas stable disease was reported in 133 patients $(73.5 \%)$ and progressive disease in 44 cases (24.3\%). Meng et al (34) concluded that MTI monotherapy may be recommended as first-line treatment. Combination therapy with two MTIs may be administered for drug-resistant chordoma.

In conclusion, extraosseous nasopharyngeal chordoma is a rare type of tumor that is amenable to surgery and postoperative radiotherapy. RapidArc VMAT is a technique that achieves excellent local control of these tumors with a greater sparing effect of normal tissues. However, additional studies are required to further elucidate the clinical behavior and determine the optimal treatment strategy for nasopharyngeal chordoma.

\section{Acknowledgements}

Not applicable.

\section{Funding}

No funding was received.

\section{Availability of data and materials}

The datasets used and/or analyzed during the current study are available from the corresponding author on reasonable request.

\section{Authors' contributions}

CHY collected all the pertinent data of the patient, wrote and edited the final manuscript.

\section{Ethics approval and consent to participate}

The study was approved by the institutional review board of the Tungs' Taichung Metroharbor Hospital (IRB no. 109033). 


\section{Patient consent for publication}

The present study was granted an exemption from requiring patient's informed consent for publication by the institutional review board of the Tungs' Taichung Metroharbor Hospital (IRB no. 109033).

\section{Competing interests}

The author declares no competing interests.

\section{References}

1. Bakker SH, Jacobs WCH, Pondaag W, Gelderblom H, Nout RA, Dijkstra PDS, Peul WC and Vleggeert-Lanamo CLA: Chordoma: A systematic review of the epidemiology and clinical prognostic factors predicting progression-free and overall survival. Eur Spine J 27: 3043-3058, 2018.

2. Hulou MM, Garcia CR, Slone SA, Dugan A, Lei F, Huang B, Pittman T and Villano JL: Comprehensive review of cranial chordomas using national databases in the USA. Clin Oncol (R Coll Radiol) 31: e149-e159, 2019

3. Cha YJ and Suh YL: Chordomas: Histopathological study in view of anatomical location. J Korean Med Sci 34: e107, 2019.

4. Kataria SP, Batra A, Singh G, Kumar S and Sen R: Chordoma of skull base presenting as nasopharyngeal mass. J Neurosci Rural Pract 4 (Suppl 1): S95-S97, 2013.

5. Vaz-Guimaraes F and Harsh IV GR: Chapter 5: Demographics, presentation, and diagnosis: Chordomas and chondrosarcomas of the skull base and spine. 2nd edition. Harsh IV GR and Vas-Guimaraes F (eds). Academic press, London, pp45-51, 2018.

6. Foweraker KL, Burton KE, Maynard SE, Jena R, Jefferies SJ, Laing RJC and Burnet NG: High-dose radiotherapy in the management of chordoma and chondrosarcoma of the skull base and cervical spine: Part 1-clinical outcomes. Clin Oncol (R Coll Radiol) 19: 509-516, 2007.

7. Goodwin CR, Liang LJ, Zadnik PL and Sciubba DM: Chapter 155: Chordomas and chondrosarcomas. In: Youmans and Winn neurological surgery. Richard WH (ed). Vol 4. 7th edition. Elsevier, Philadelphia, PA, pp1243-1250, 2017.

8. Windeyer BW: Chordoma. Proc R Soc Med 52: 1088-1100, 1959

9. Kerr DA and Rosenberg AE: Chapter 2: Pathology of chordoma and chondrosarcoma of the axial skeleton. In: Chordomas and chondrosarcomas of the skull base and spine. Harsh IV GR and Vas-Guimaraes F (eds). 2nd edition. Academic press, London, pp11-21, 2018.

10. Burrow Jle F and Stewart MJ: Original papers: Malignant spheno-occipital chordoma. J Neurol Psychopathol 4 205-217,1923.

11. Seltzer AP: Nasopharyngeal chordoma. A case report. J Natl Med Assoc 53: 41-42, 1961.

12. Nguyen RP, Salzman KL, Stambuk HE, Ahuja AT and Harnsberger HR: Extraosseous chordoma of the nasopharynx. AJNR Am J Neuroradiol 30: 803-807, 2009.

13. Vasudevan V, Manjunath V, Devaraju D and Murali R: Chordoma arising from cranial base extending to oropharynx: An unusual presentation. J Maxillofac Oral Surg 14 (Suppl 1): S103-S107, 2015.

14. Jallo J, Nathan D, Bierbrauer K and Farber E: Chordoma: A case report. Surg Neurol 48: 46-48, 1997.

15. Kneisl JS, Rosenberg AE, Anderson PM, Antonescu CR, Bruland OS, Cooper K, Horvai AE, Holt GE, O'Sullivan B, Patel SR, et al: Chapter 38: Bone. In: AJCC cancer staging manual. Amin MB, Edge S, Greene F, Byrd DR, Brookland RK, Washington MK, Gershenwald JE, Compton CC, Hess KR, Sullivan DC, et al (eds). 8th edition. Springer International Publishing, New York, NY, pp471-486, 2017.

16. Guo R, Tang LL, Mao YP, Zhou GQ, Qi ZY, Liu LZ, Lin AH Liu MZ, Ma J and Sun Y: Clinical outcomes of volume-modulated arc therapy in 205 Patients with nasopharyngeal carcinoma: An analysis of survival and treatment toxicities. PLoS One 10 e0129679, 2015.
17. Wasserman JK, Gravel D and Purgina B: Chordoma of the head and neck: A review. Head Neck Pathol 12: 261-268, 2018.

18. McKeever PE and Venneti S: Chapter 20: Immunohistology of the nervous system. In: Diagnostic immunohistochemistry, theranostic and genomic applications. Dabbs DJ (ed). 5th edition. Elsevier, Inc., Philadelphia, PA, pp824-826, 2019.

19. Mohyeldin A, Vaz-Guimaraes F, Carrau RL and Prevedello DM: Chapter 3: Molecular drivers in chordoma. In: Chordomas and chondrosarcomas of the skull base and spine. Harsh IV GR and Vas-Guimaraes F (eds). 2nd edition. Academic press, London, pp23-29, 2018.

20. Mitra B, Sengupta S, Rai A, Mehta J, Quader AR, Roy S and Borges A: Chordoma in nasopharynx in a 70-year-old female: A rare occurrence. Int J Otolaryngol Head Neck Surg 3: 342-346, 2014.

21. Sajisevi M, Hoang JK, Eapen R and Jang DW: Nasopharyngeal masses arising from embryologic remnants of the clivus: A case series. J Neurol Surg Rep 76: e253-e257, 2015.

22. Salazar Guilarte JX, Sancho Mestre M and Gras Albert JR: Extraosseous chordoma of nasopharynx in pediatric age. Acta Otorrinolaringol Esp 63: 321-323, 2012 (In Spanish).

23. Orecchia R, Leonardi MC, Krengli M, Zurrida S and Brambilla MG: External radiotherapy plus intracavitary brachytherapy for recurrent chordoma of the nasopharynx. Acta Oncol 37: 301-304, 1998.

24. Walcott BP, Nahed BV, Mohyeldin A, Coumans JV, Kahle KT and Ferreira MJ: Chordoma: Current concepts, management, and future directions. Lancet Oncol 13: e69-e76, 2012

25. Radzikowska J, Gronkiewicz Z, Kukwa A, Lisik W, Czarnecka AM, Krzeski A and Kukwa W: Nasopharyngeal chordoma in a patient with a severe form of sleep-disordered breathing: A case report. Oncol Lett 10: 1805-1809, 2015.

26. Dwianingsih EK, Snak Y, Rinonce HT, Wasita B, Antoro EL and Amr SS: Primary chordoma of the nasopharynx: A rare case report and review of the literatures. Case Rep Pathol 2019: $3826521,2019$.

27. Young VA, Curtis KM, Temple HT, Eismont FJ, DeLaney TF and Hornicek FJ: Characteristics and patterns of metastatic disease from chordoma. Sarcoma 2015: 517657, 2015.

28. Zhang HK, Sun XC, Hu L, Wang JJ and Wang DH: Endonasal endoscopic resection and radiotherapy in skull base chordomas. J Craniofac Surg 27: e709-e713, 2016.

29. Teoh M, Clark CH, Wood K, Whitaker S and Nisbet A: Volumetric modulated arc therapy: A review of current literature and clinical use in practice. Br J Radiol 84: 967-996, 2011

30. Sahgal A, Chan MW, Atenafu EG, Masson-Cote L, Bahl G, Yu E, Millar BA, Chung C, Catton C, O'Sullivan B, et al: Image-guided, intensity-modulated radiation therapy (IG-IMRT) for skull base chordoma and chondrosarcoma: Preliminary outcomes. Neuro Oncol 17: 889-894, 2015.

31. Gatfield ER, Noble DJ, Barnett GC, Early NY, Hoole ACF, Kirkby NF, Jefferies SJ and Burnet NG: Tumor volume and dose influence outcome after surgery and high-dose photon radiotherapy for chordoma and chondrosarcoma of the skull base and spine. Clin Oncol (R Coll Radiol) 30: 243-253, 2018.

32. Stacchiotti S, Gronchi A, Fossati P, Akiyama T, Alapetite C, Baumann M, Blay JY, Bolle S, Boriani S, Bruzzi P, et al: Best practices for the management of local-regional recurrent chordoma: A position paper by the chordoma global consensus group. Ann Oncol 28: 1230-1242, 2017.

33. Takagi M, Demizu Y, Nagano F, Terashima K, Fujii O, Jin D, Mima M, Niwa Y, Katsui K, Suga M, et al: Treatment outcomes of proton or carbon ion therapy for skull base chordoma: A retrospective study. Radiat Oncol 13: 232, 2018.

34. Meng T, Jin J, Jiang C, Huang R, Yin H, Song D and Cheng L: Molecular targeted therapy in the treatment of chordoma: A systematic review. Front Oncol 9: 30, 2019.

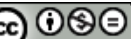

This work is licensed under a Creative Commons Attribution-NonCommercial-NoDerivatives 4.0 International (CC BY-NC-ND 4.0) License. 